\title{
On the potassium-rotation connection in late-type Alpha Persei stars ${ }^{\star}$
}

\author{
E. L. Martín ${ }^{1,2}$, A. Magazzù ${ }^{3}$, R. J. García López ${ }^{1}$, S. Randich ${ }^{4}$, and D. Barrado y Navascués ${ }^{5}$ \\ 1 Instituto de Astrofísica de Canarias, 38200 La Laguna, Spain \\ e-mail: ege@iac.es \\ 2 University of Central Florida, Department of Physics, PO Box 162385, Orlando, FL 32816-2385, USA \\ e-mail: ege@physics.ucf.edu \\ 3 Centro Galileo Galilei, Apartado 565, 38700 Santa Cruz de La Palma, Spain \\ e-mail: magazzu@tng.iac.es \\ 4 Osservatorio Astrofisico de Arcetri, Largo Fermi 5, 50125 Firenze, Italy \\ e-mail: randich@arcetri.astro.it \\ 5 Laboratorio de Astrofísica Espacial y Física Fundamental, INTA, Apartado Postal 50727, 28080 Madrid, Spain \\ e-mail: barrado@laeff.esa.es
}

Received 9 July 2004 / Accepted 14 September 2004

\begin{abstract}
We present measurements of the K I $\lambda 7699$ line from spectra of 19 late-type members of the $\alpha$ Persei cluster obtained with the Intermediate Dispersion Spectrograph at the Isaac Newton Telescope. These stars span a narrow range of $T_{\text {eff }}$, from $5091 \mathrm{~K}$ to $4771 \mathrm{~K}$, and a wide range of $v \sin i$ values $\left(9 \mathrm{~km} \mathrm{~s}^{-1}-170 \mathrm{~km} \mathrm{~s}^{-1}\right)$. For a given star, we find empirically that larger rotational broadening apparently increases the equivalent width of K I $\lambda 7699$ linearly for $v \sin i$ values from $15 \mathrm{~km} \mathrm{~s}^{-1}$ to $75 \mathrm{~km} \mathrm{~s}^{-1}$. This correlation breaks down for $v \sin i>75 \mathrm{~km} \mathrm{~s}^{-1}$. After correction for this effect, we show that the potassium line equivalent widths do not really correlate with $v \sin i$.
\end{abstract}

Key words. line: formation - stars: atmospheres - stars: abundances - stars: activity - stars: late-type Galaxy: open clusters and associations: alpha Per

\section{Introduction}

A long standing problem in stellar evolution has been the connection between lithium abundances and rotation in low-mass young stars (Soderblom et al. 1993; García López et al. 1994). Lithium can be destroyed inside stars, and a spread in Li I line strengths could be interpreted as evidence for a role of rotation in Li depletion. Such a role cannot be explained with standard models of lithium depletion, simply because they do not include rotation. Modifications of the standard model include the effect of the rotational history among stars with the same age and mass (Chaboyer et al. 1995; Martín \& Claret 1996), magnetic fields at the base of the convection zone (Ventura et al. 1998) and gravity waves (Montalban \& Schatzman 1996).

An alternative interpretation of the lithium-rotation connection is that it is not due to differences in the lithium abundances, but to effects of rotation on the formation of the lithium resonance line. Careful analysis of the subordinate lithium line

^ Based on observations made with the Isaac Newton Telescope, operated on the island of La Palma by the Isaac Newton Group in the Spanish Observatorio del Roque de los Muchachos of the Instituto de Astrofísica de Canarias. at $610.4 \mathrm{~nm}$ by Ford, Jeffries \& Smalley (2002) indicates that the spread in lithium abundances among Pleiades stars with the same mass may be real.

Dispersion of K I $\lambda 7699$ equivalent width measurements in Pleiades low-mass stars has been reported by Soderblom et al. (1993) and Jeffries (1999). For a given effective temperature, stars with high chromospheric activity and fast rotation tend to have stronger K I lines. The Li I and K I lines have similar formation mechanisms (Stuik et al. 1997). Since potassium is not destroyed in stellar interiors, all cluster members are expected to have the same abundance.

The spread in KI line strength suggests that the dispersion in Li I may not be due to abundance differences among the stars, but to the formation process of the lines. Active stars are known to have surface spots where the Li I and KI lines are stronger. However, monitoring of the variability of these lines in active stars in the Pleiades has failed to provide conclusive evidence for spot-induced modulation (Patterer et al. 1993; Jeffries 1999). Possible variability on timescales of years has been noted (Martín \& Claret 1996), which could be due to cycles of magnetic activity, but this has not been demonstrated 
with long-term monitoring. The spread in $\mathrm{K}$ I equivalent widths for stars of similar $T_{\text {eff }}$ in the Pleiades cluster remains so far unaccounted for.

Randich et al. (1998) found a similar behaviour for Li I in cool stars of the Alpha Persei open cluster as in their Pleiades counterparts. We present here KI equivalent width measurements in Alpha Persei stars from Randich et al. (1998), with the aim to test whether in these objects potassium shows a similar spread as lithium. In Sect. 2 we describe our observations. In Sect. 3 we give an analysis of the effect of rotational broadening on the measurement of equivalent widths. We find that the potassium-rotation connection dissapears when the equivalent width measurements are corrected for the effect of rotation on the line profiles. In Sect. 4 we discuss these results. We conclude that the potassium-rotation connection in late-type members of young open clusters may largely be due to the effect of rotational broadening in the measurement of equivalent widths.

\section{Observations}

Objects of our sample have been selected from Randich et al. (1998). They are listed in Table 1, together with their effective temperatures and rotational velocities. We chose stars within a narrow range of $T_{\text {eff }}$ and spanning a wide range of rotational broadening. Optical spectra were obtained with the Intermediate Dispersion Spectrograph at the Cassegrain focus of the $2.5 \mathrm{~m}$ Isaac Newton Telescope in La Palma. The observations were performed in the night of November 7, 2000. The $235 \mathrm{~mm}$ camera, equipped with a EEV CCD, was used in conjunction with the grating $\mathrm{H} 1800 \mathrm{~V}$. A resolution of $\sim 1 \AA$ was achieved. Exposure times of $1200 \mathrm{~s}$ were used for all the spectra, except in the case of the object AP43, which was observed with an exposure time of $1600 \mathrm{~s}$. Data reduction, including bias subtraction, flat-fielding, extraction of one-dimensional spectra, and wavelength calibration, was carried out using the IRAF $^{1}$ environment.

Equivalent widths of the K I $\lambda 7699$ line have been measured by direct integration and are reported in Table 1 . The average $3 \sigma$ error bar is $10 \%$ of the equivalent width. For AP33 and AP70 the $3 \sigma$ error bar is $20 \%$. Figure 1 shows these measurements vs. $v \sin i$ (dots), together with data obtained in the Pleiades by Jeffries (1999; open squares) and Soderblom et al. (1993; crosses). This figure indicates a large spread in equivalent widths, with a trend for stars in the Alpha Persei cluster with higher $v \sin i$ also to also have a stronger $\mathrm{KI}$ line, consistently with previous results in the Pleiades. It seems that all stars with $v \sin i$ larger than $30 \mathrm{~km} \mathrm{~s}^{-1}$ in these two young open clusters have systematically larger K I $\lambda 7699$ lines than their counterparts with slower rotation rates. This systematic effect is significant given the error bars in the equivalent widths $(\leq 10 \%)$. However, in the next section we will show that the correlation between $\mathrm{K} \mathrm{I}$ line and $v \sin i$ is spurious.

It is well known that the rotational broadening of a spectrum changes the line profiles and appearance of the

\footnotetext{
${ }^{1}$ IRAF is distributed by the National Optical Astronomy Observatory, which is operated by the Association of Universities for Research in Astronomy, Inc., under contract with the National Science Foundation.
}

Table 1. Our sample.

\begin{tabular}{llll}
\hline \hline Star & $\begin{array}{l}T_{\text {eff }} \\
(\mathrm{K})\end{array}$ & $\begin{array}{l}v \sin i \\
\left(\mathrm{~km} \mathrm{~s}^{-1}\right)\end{array}$ & $\begin{array}{l}W_{\lambda} \mathrm{K} \mathrm{I} \\
(\AA)\end{array}$ \\
\hline AP33 & 4836 & 9 & 0.38 \\
AP37 & 4977 & 29 & 0.34 \\
AP43 & 4840 & 72 & 0.43 \\
AP56 & 4801 & 110 & 0.41 \\
AP65 & 4798 & 10 & 0.35 \\
AP70 & 4886 & 9 & 0.35 \\
AP72 & 4911 & 9 & 0.31 \\
AP78 & 4775 & 13 & 0.46 \\
AP91 & 4837 & 25 & 0.40 \\
AP93 & 4873 & 75 & 0.50 \\
AP98 & 4912 & 9 & 0.37 \\
AP106 & 4847 & 9 & 0.33 \\
AP110 & 5087 & 9 & 0.33 \\
APX1 & 5091 & 13 & 0.33 \\
APX27A & 5009 & 50 & 0.45 \\
APX49A & 4795 & 21 & 0.32 \\
APX59 & 4957 & 38 & 0.34 \\
APX72 & 4893 & 16 & 0.32 \\
APX158 & 5001 & 170 & 0.47 \\
\hline
\end{tabular}

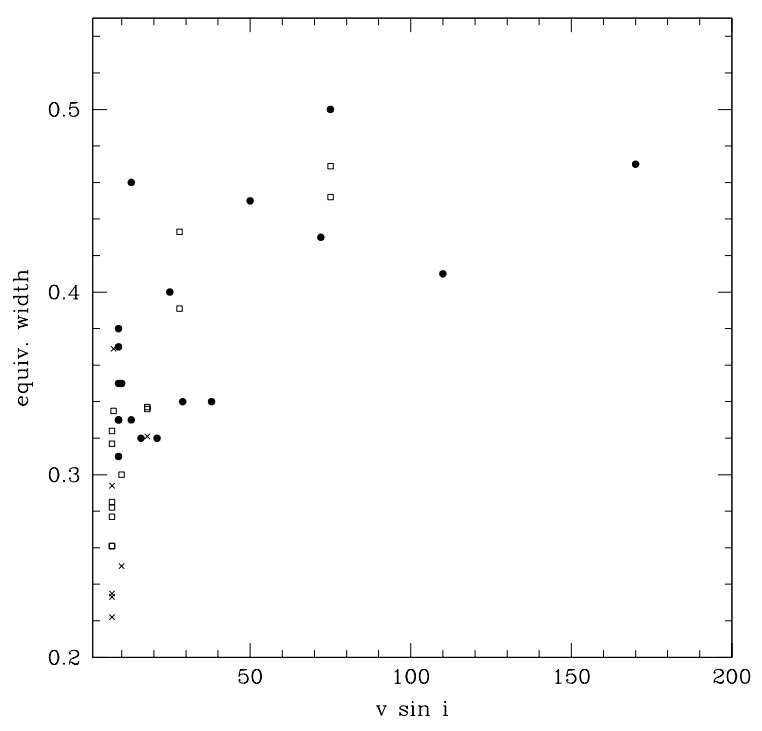

Fig. 1. Equivalent widths of the K I $\lambda 7699$ line versus rotation velocity. Different symbols denote different samples as follows: our Alpha Persei stars (filled dots), Pleiades stars from Jeffries (1999) (open squares) and Pleiades stars from Soderblom et al. (1993) (crosses). Only stars with $T_{\text {eff }}$ in the range 5091-4771 $\mathrm{K}$ are shown.

continuum. This can also affect the equivalent width measurements. Hence, it is not possible to use the same continuum regions for all stars in the sample, regardless of their rotational broadening. In the next section we develop a method to correct the K I equivalent widths from the effect of rotational broadening.

\section{Analysis}

\subsection{Estimating the effects of rotational broadening}

We broadened the spectra of slow rotators with rotational profiles of various $v \sin i$ 's, measured the equivalent width of the 
Table 2. Reference stars for fast rotators.

\begin{tabular}{lll}
\hline \hline Fast rotator & Reference star & $\begin{array}{l}\Delta T_{\text {eff }} \\
(\mathrm{K})\end{array}$ \\
\hline AP37 & $\begin{cases}\text { AP98 } \\
\text { AP72 }\end{cases}$ & +65 \\
AP43 & $\begin{cases}\text { AP106 } \\
\text { AP33 }\end{cases}$ & -7 \\
AP56 & $\begin{cases}\text { AP65 } \\
\text { AP33 }\end{cases}$ \\
AP91 & $\begin{cases}\text { AP33 } \\
\text { AP110 }\end{cases}$ \\
AP93 & $\begin{cases}\text { AP70 } \\
\text { AP106 }\end{cases}$ \\
APX27A & $\begin{cases}\text { AP110 } \\
\text { AP98 }\end{cases}$ \\
APX59 & $\begin{cases}\text { AP98 } \\
\text { AP72 }\end{cases}$ \\
APX158 & $\begin{cases}\text { AP110 } \\
\text { AP98 }\end{cases}$ \\
\hline
\end{tabular}

Table 3. Selected reference stars and broadening velocities.

\begin{tabular}{lllll}
\hline \hline Star & \multicolumn{4}{c}{$\begin{array}{c}v \sin i \\
\left(\mathrm{~km} \mathrm{~s}^{-1}\right)\end{array}$} \\
\hline AP65 & 110 & & & \\
AP72 & 29 & 38 & & \\
AP98 & 29 & 38 & 50 & 170 \\
AP106 & 72 & 75 & & \\
AP110 & 25 & 50 & 170 & \\
\hline
\end{tabular}

K I line at different velocities, and then checked whether such equivalent widths change with $v \sin i$ in a significant way. In an attempt to make our test as realistic as possible, for each fast rotator in our sample we selected low $v \sin i$ "reference" stars in the same sample, according to similarity of the effective temperature. In Table 2 we show the matches made according to this criterion. The temperature difference between the fast rotator and reference star is reported as $\Delta T_{\text {eff. }}$. From the table we see that for each fast rotator two reference slow rotators have been found with differences in $T_{\text {eff }}$ not exceeding $100 \mathrm{~K}$ (average absolute value $\sim 33 \mathrm{~K}$ ). After the exclusion of AP33 and AP70, owing to the low signal-to-noise ratio of their spectra, we are left with five slow rotators which can be used as reference. In Table 3 we report the rotational velocities of the fast rotators matching these objects in effective temperature.

A Fortran program written by Ya. V. Pavlenko (2001, private communication) was used to broaden the spectra of stars in Table 3 by convolution with rotational profiles of $v \sin i$ 's reported in the same table. In Fig. 2 we plot a region around the K I $\lambda 7699$ line. The spectrum of AP110, broadened for several rotational velocities, is shown.

Equivalent widths of the K I $\lambda 7699$ line in the spectra of the reference stars in Table 3 have been measured by direct integration and, when possible, by gaussian fitting, using tasks within IRAF. After broadening, gaussian noise was added. Then, equivalent widths were measured in the broadened spectra by direct integration of the line profiles, in the same way as

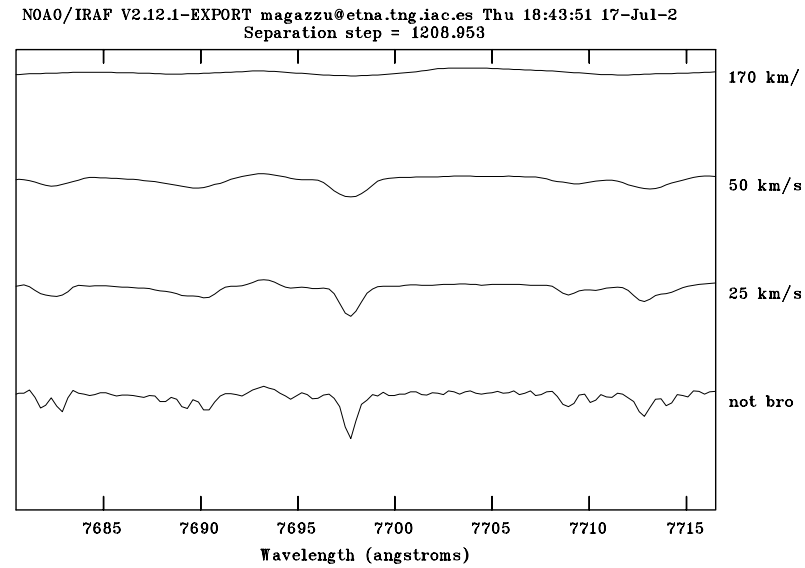

Fig. 2. The K I $\lambda 7699$ region of AP110. From bottom to top: the spectrum "as it is", and after convolution with rotational profiles $(v \sin i=$ 25,50 , and $170 \mathrm{~km} \mathrm{~s}^{-1}$ ).

the measurements performed in the original spectra. The results are shown in Table 4.

For each star in Table 4 we can calculate $W / W_{0}$, the ratio between the equivalent width at a given rotational velocity and the equivalent width in the original spectrum. This quantity is plotted vs. $v \sin i$ in Fig. 3. In this figure we see that, while $W / W_{0}$ increases for $15 \mathrm{~km} \mathrm{~s}^{-1} \lesssim v \sin i \lesssim 75 \mathrm{~km} \mathrm{~s}^{-1}$, at $100 \mathrm{~km} \mathrm{~s}^{-1}$ a small decrease is seen. It is not clear whether at higher $v \sin i$ 's there is a further decrease or if $W / W_{0}$ can be considered constant for $v \sin i \gtrsim 100 \mathrm{~km} \mathrm{~s}^{-1}$. We can explain this trend by considering that an increase of the broadening leads also to an increase of the blending of the K I line with adjacent lines, with as a consequence the enlargement of the measured equivalent width. However, for higher velocities, the lines become so shallow that the integration extremes are difficult to define, which will tend to underestimate the integration range. Therefore, a fraction of flux does not contribute to the equivalent width, somewhat compensating for the blending. The velocity corresponding to the breakdown of the linear increase depends on the resolution of the data.

In any case, Fig. 3 indicates a significant increase of the equivalent width due to rotational broadening. Data in Fig. 3 fit a very tight linear relationship (correlation factor $=0.996$ ) for $15 \mathrm{~km} \mathrm{~s}^{-1}<v \sin i<75 \mathrm{~km} \mathrm{~s}^{-1}$. For faster velocities, we suppose a constant behaviour and set $W / W_{0}$ equal to the average of the three highest velocity points in our sample. For $v \sin i<15 \mathrm{~km} \mathrm{~s}^{-1}$ we cannot calibrate the effect of rotation because of the modest resolution of our spectra. In summary:

$W / W_{0}=\left\{\begin{array}{l}0.89+5.14 \times 10^{-3} \cdot v \sin i \\ \text { if } 15 \mathrm{~km} \mathrm{~s}^{-1}<v \sin i<75 \mathrm{~km} \mathrm{~s}^{-1} \\ 1.17 \text { if } v \sin i>75 \mathrm{~km} \mathrm{~s}^{-1}\end{array}\right.$

\subsection{Correcting our equivalent widths}

We use these results to correct our measurements. According to Eq. (1), we determine $W / W_{0}$ for each of the stars observed in this work and divide the equivalent widths in Table 1 by this quantity. This allows us to correct our equivalent widths 
Table 4. K I $\lambda 7699$ equivalent widths in the spectra of slow rotators broadened for various velocities.

\begin{tabular}{|c|c|c|c|c|c|c|c|c|c|}
\hline \multirow[t]{2}{*}{ Star } & \multirow[b]{2}{*}{$v \sin i\left(\mathrm{~km} \mathrm{~s}^{-1}\right)$} & \multicolumn{8}{|c|}{$W_{\lambda}(\AA)$} \\
\hline & & 25 & 29 & 38 & 50 & 72 & 75 & 110 & 170 \\
\hline AP65 & & & & & & & & 0.41 & \\
\hline AP72 & & & 0.33 & 0.35 & & & & & \\
\hline AP98 & & & 0.38 & & 0.42 & & & & 0.41 \\
\hline AP106 & & & & & & 0.41 & 0.42 & & \\
\hline AP110 & & 0.33 & & & 0.37 & & & & 0.39 \\
\hline
\end{tabular}

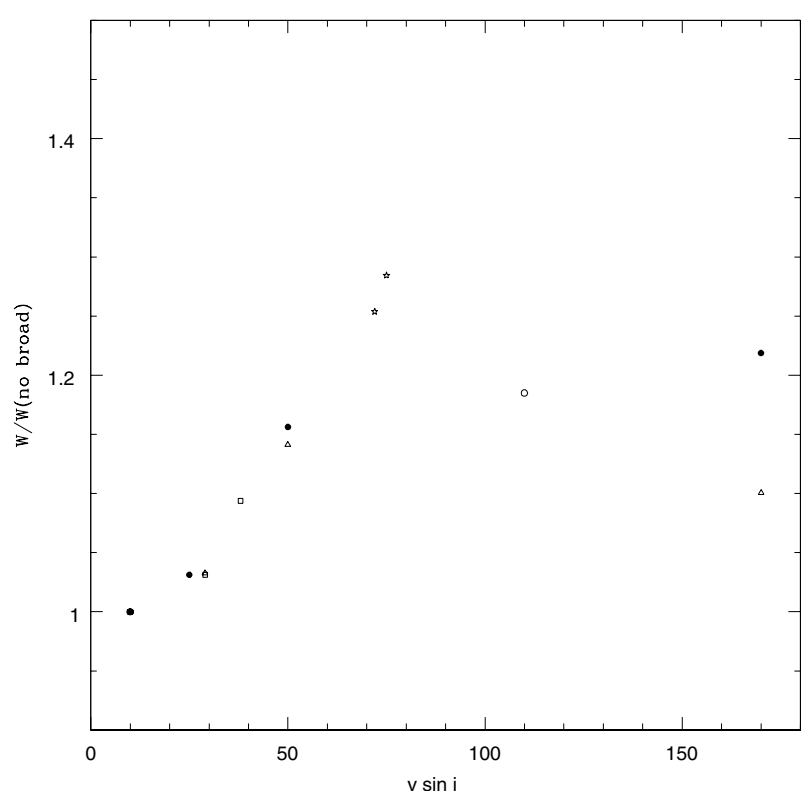

Fig. 3. $W / W_{0}$ vs. $v \sin i$.

Table 5. Corrected equivalent widths.

\begin{tabular}{ll}
\hline \hline Star & $\begin{array}{l}W_{\lambda} \\
(\AA)\end{array}$ \\
\hline AP37 & 0.34 \\
AP43 & 0.43 \\
AP56 & 0.41 \\
AP91 & 0.40 \\
AP93 & 0.50 \\
APX27A & 0.45 \\
APX49A & 0.32 \\
APX59 & 0.34 \\
APX72 & 0.32 \\
APX158 & 0.47 \\
\hline
\end{tabular}

for the effects of rotational broadening. Final equivalent widths are listed in Table 5 and shown vs. $v \sin i$ in Fig. 4.

\section{Discussion and perspectives}

A look at Fig. 4 suggests no significant correlation between $\mathrm{KI}$ equivalent widths and $v \sin i$. This is corroborated by the poor correlation factor, equal to 0.26 . Note that the corresponding correlation factor for data in Table 1 (equivalent widths before correction) is 0.64 . We see also that the average corrected equivalent width for stars with $v \sin i \leq 15 \mathrm{~km} \mathrm{~s}^{-1}$

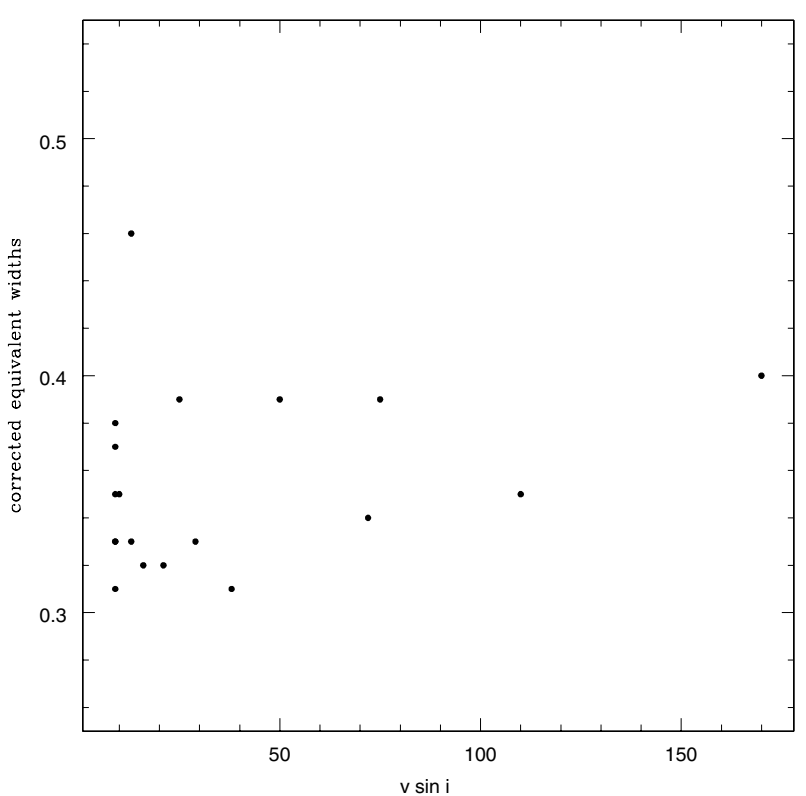

Fig. 4. Corrected equivalent widths vs. $v \sin i$.

is $0.36 \pm 0.4 \AA$, practically the same as for faster rotators $(0.35 \pm 0.4 \AA)$.

Thus, in our Alpha Persei sample, the faster rotators do not show stronger K I $\lambda 7699$ lines than the slow rotators. The increase of equivalent width with $v \sin i$ can be explained in terms of a systematic effect due to rotational broadening. The effect found in this work could also be present in other data sets of similar resolution. This could also explain the trend seen in the Pleiades (Soderblom et al. 1993; Jeffries 1999).

There is still a significant spread in the KI equivalent widths shown in Fig. 4. It seems that the effect of $v \sin i$ on the measurement of equivalent widths does not explain completely the scatter in the line strengths. Other effects may be present that change the formation of the lines, but they do not depend on rotation. Possibly the spread is caused by small differences in $T_{\text {eff }}$ among the stars in our sample because the line is very sensitive to temperature. For example, the star with the largest K I equivalent width (AP78) in Fig. 4 is also the coolest star included in our study $\left(T_{\text {eff }}=4775 \mathrm{~K}\right)$. Another factor that could play a role in the spread of corrected $\mathrm{K} \mathrm{I}$ equivalent widths is stellar activity because of the effect of stellar spots and faculae on the line formation (Barrado y Navascués et al. 2001).

Since the K I $\lambda 7699$ line is considered to be a good proxy of the Li I $\lambda 6708$ line, we plan to perform a similar test of the dependence of the Li I equivalent width on $v \sin i$ in a future 
work. The assumption that the equivalent widths of individual lines is invariant for different values of $v \sin i$ may need to be revised.

\section{References}

Barrado y Navascués, D., García López, R. J., Severino, G., \& Gomez, M. T. 2001, A\&A, 371, 652

Chaboyer, B., Demarque, P., \& Pinsonneault, M. H. 1995, ApJ, 441, 876

Ford, A., Jeffries, R. D., \& Smalley, B. 2002, A\&A, 391, 253

García López, R. J., Rebolo, R., \& Martín, E. L. 1994, A\&A, 282, 518
Jeffries, R. D., 1999, MNRAS, 309, 189

Martín, E. L., \& Claret, A. 1996, A\&A, 306, 408

Montalban, J., \& Schatzman, E. 1996, A\&A, 305, 513

Patterer, R. J., Ramsey, L., Huenemoerder, D. P., \& Welty, A. D. 1993, AJ, 115, 1519

Randich, S., Martín, E. L., García López, R. J., \& Pallavicini, R. 1998, A\&A, 333, 591

Soderblom, D. R., Jones, B. F., Balachandran, S., et al. 1993, AJ, 106, 1059

Stuik, R., Bruls, J. H. M. J., \& Rutten, R. J. 1997, A\&A, 332, 911

Ventura, P., Zeppieri, A., Mazzitelli, I., \& D’Antona, F. 1998, A\&A, 331,1011 